\title{
Phase-differential NIR integral field spectroscopy of transiting Hot Jupiters
}

\section{Daniel Angerhausen, Alfred Krabbe and Christof Iserlohe}

Physikalisches Institut, Universität zu Köln, Zülpicher Strasse 77, 50937 Köln, Germany email: anger@ph1.uni-koeln.de

\begin{abstract}
Transiting exoplanets provide a unique opportunity for follow up exploration through phase-differential observation of their emission and transmission spectra. From such spectra immediate clues about the atmospheric composition and the planets chemistry can be drawn. Such information is of imminent importance for the theory of the formation of planets in general as well as for their particular evolution. Ground-based spectroscopy of exoplanet transits is a needful extension of results already obtained through space-based observations. We present results of an exploratory study to use near-infrared integral field spectroscopy to observe extrasolar planets. We demonstrate how adaptive optics-assisted integral field spectroscopy compares with other spectroscopic techniques currently applied. An advanced reduction method using elements of a spectral-differential decorrelation method is also discussed. We have tested our concept with a K-Band time series observations of HD209458b and HD189733b obtained with SINFONI at the VLT and OSIRIS at Keck during secondary transits at a spectral resolution of $\mathrm{R}=3000$.
\end{abstract}

\section{Introduction}

Observations of extrasolar planets are always limited by the contribution of their parent stars. While Hot Jupiters for example show a reasonable IR-contrast ratio, they are very close to their parent star and spatially not resolvable with present telescopes. To separate the planetary signal, methods are needed that cancel out or suppress the stellar contribution.

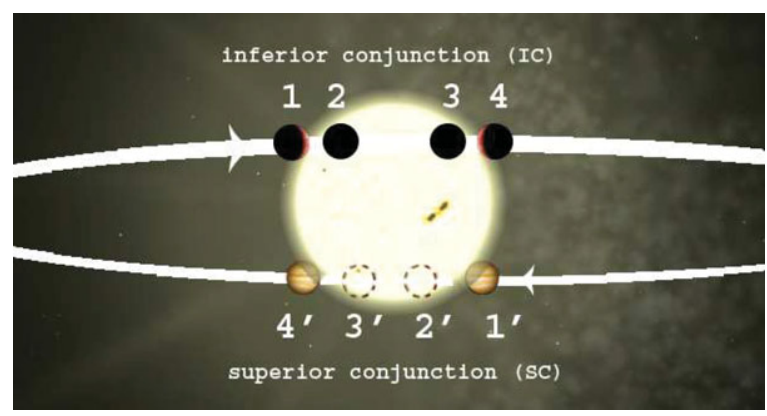

Figure 1. The secondary eclipse or superior conjunction is the moment the planet orbits behind the host star. The difference between the stars spectrum in this phase and the spectrum after the recurrence of the planet reveals the planetary spectrum.

Detailed models of contrast and expected signal-to-noise ratio show that a subgroup of transiting exoplanets is already observable with near infrared integral field spectroscopy from large ground based telescopes. The phases of conjuntions provide the opportunity to obtain planetary spectra by comparing the spectra observed during the different phases 
(Fig 1). The best of these candidates so far are the transiting Hot Jupiter exoplanets HD 209458b and HD 189733b.

\section{Adaptive Optics assisted imaging spectroscopy}

Using integral field units (Fig. 2) like SINFONI at the VLT or OSIRIS at Keck in AO mode has several advantages for spectrocopy of extrasolar planets:

- Systematic noise is minimized

- 2 to 3 times more sensitive than single slit spectroscopy

- Maximum observing efficiency

- Smaller scale lowers thermal background in K-band

- Time scale of spatial modulations reduced

- Dithering steps are much better controllable
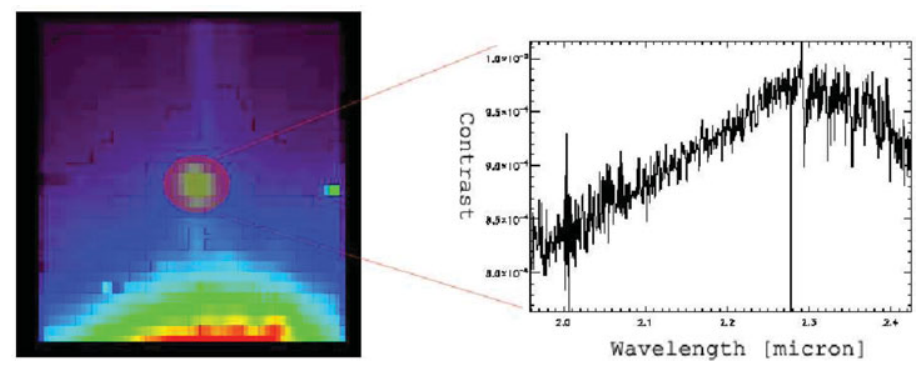

Figure 2. Example of a SINFONI observation of GQ Lup b (ESO Archive). Left: Wavelength-collapsed image of star (bottom) and companion (center). Right: extracted spectrum of companion. Imaging spectroscopy offers the opportunity to extract a spectrum of every pixel in the 2D-field of view.

\section{Observations}

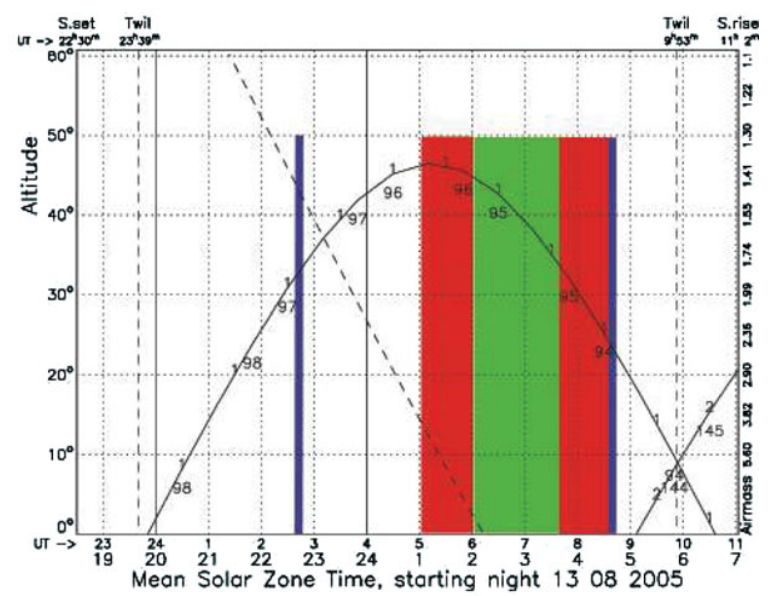

Figure 3. Schedule of the observation. Blue lines represent start and end of our observation. The green area marks the phase of certain conjunction, red areas represent phases of ingress and egress. In and out of eclipse data can be observed at the same elevation/airmass.

HD 209458b was observed before and during its secondary eclipse on August, 13th, 2005 (Fig 3). During 6 hours of DDT-Observation under slightly variable conditions we 
obtained 38 minutes of out of eclipse and 60 minutes in eclipse data. Analogous observations of HD 189733b under better conditions with an optimized strategy have been conducted with OSIRIS at KeckII in July 2007 and SINFONI at VLT in August/September 2007.

\section{Data Reduction Concept}

The challenge of the data reduction was to cope with the variable atmospheric conditions during the observations. After reducing the obtained data to 3d- data cubes, using the standard reduction pipeline, spectra were extracted slice by slice by integrating over an aperture around the fitted PSF peaks. Wavelength shifts between the individual spectra were corrected by correlating the stellar Brackett-gamma lines in every single spectrum. Gross transmission of each spectrum was corrected by applying a fit to the timeseries of spectral intensities in spectral parts with more than 97 percent of atmospheric transmission.
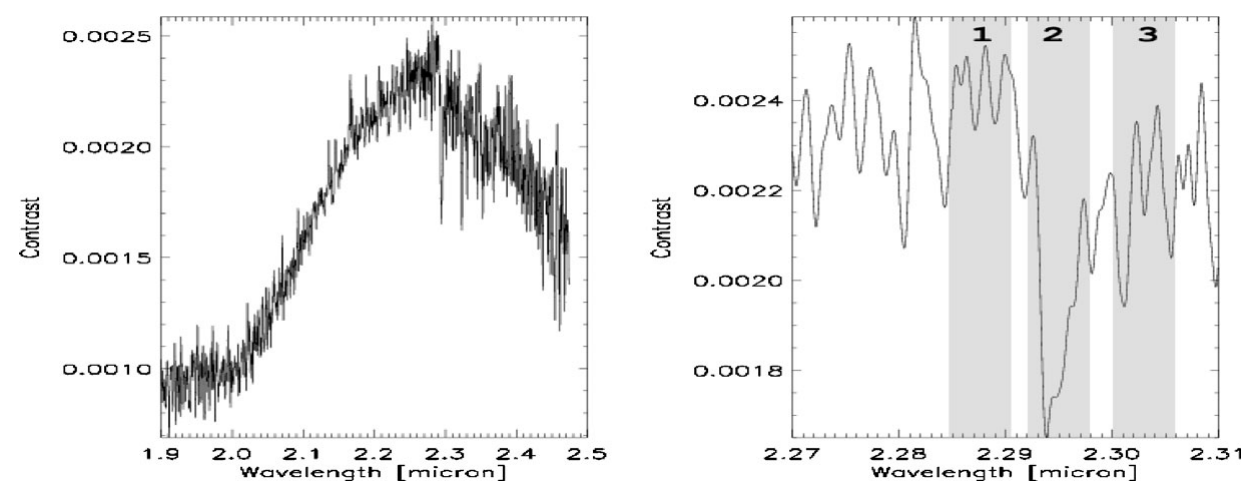

Figure 4. Hot Jupiter model spectrum by Barman (2005). Left: K-Band contrast spectrum for HD 189733b. For analysis of the broad band spectrum pairs of in and out of eclipse spectra taken at the same airmass were compared. Right: Narrow band CO-feature at $2.29 \mathrm{mi}-$ cron, grey areas and numbers highlight the wavelength ranges used to define the spectral index $I_{s}=\left(F_{1}-F_{2}+F_{3}\right) /\left(F_{1}+F_{2}+F_{3}\right)$. A timeseries of this index was analysed in a spectral differential and decorrelation method.

After the standard reduction steps two different methods were used to analyse the broad band planetary spectrum and narrow band spectral features (Fig. 4) respectively. For the analysis of the broad band spectrum pairs of in and out of eclipse spectra observed at the same airmass were divided. Averaging those contrast spectra leads to a final broad band spectrum. For analysis of narrow band features a spectral index $I_{s}=\left(F_{1}-F_{2}+F_{3}\right) /$ $\left(F_{1}+F_{2}+F_{3}\right)$ was defined (Fig. 4 , left). The index was decorrelated with observational parameters such as airmass, seeing, humidity or local temperature (Arribas 2006). Each decorrelation step slightly improved the standard deviation of the lightcurve (Fig. 5).

\section{Conclusions}

The final broad band spectrum is displayed in Figure 6 (left). Its overall shape is still dominated by systematic noise, mostly due to temporal changes in atmospheric trace gases. Since narrow band noise compares well with the calculations, spectral lines and narrow-band features are probably not dominated by systematic effects.

The lightcurve of the spectral index of the 2.3-micron feature was decorrelated with all available observational parameters. The same method was applied to an artificial 


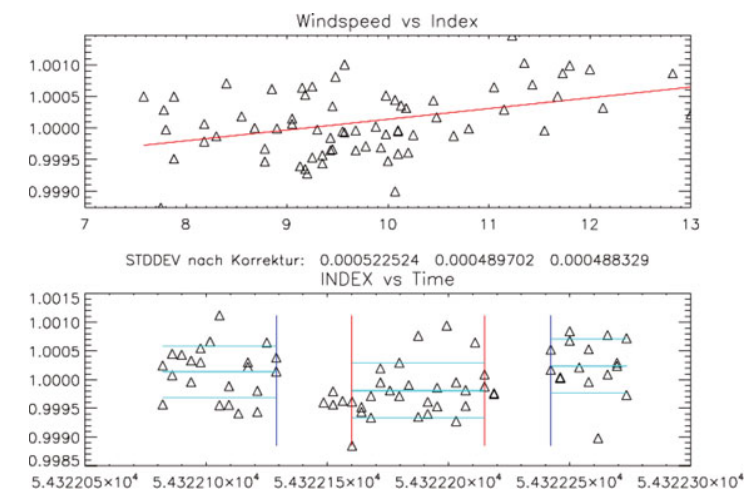

Figure 5. Example of one decorrelation step. Top: The spectral index is plotted against the observational parameter windspeed and decorrelated by a linear fit. Bottom: time-series of spectral index with improved standard deviation after decorrelation (between the red lines: in eclipse, left and right: out of eclipse).

dataset with a simulated planetary signal added to the out of eclipse spectra. Preliminary results are shown in Figure 6 (right). Our model calculations and the result of this work demonstrate the feasibility of the described spectroscopic observations. However, problems concerning changes in atmospheric trace gases and the optimization of the decorrelation method need to be solved.
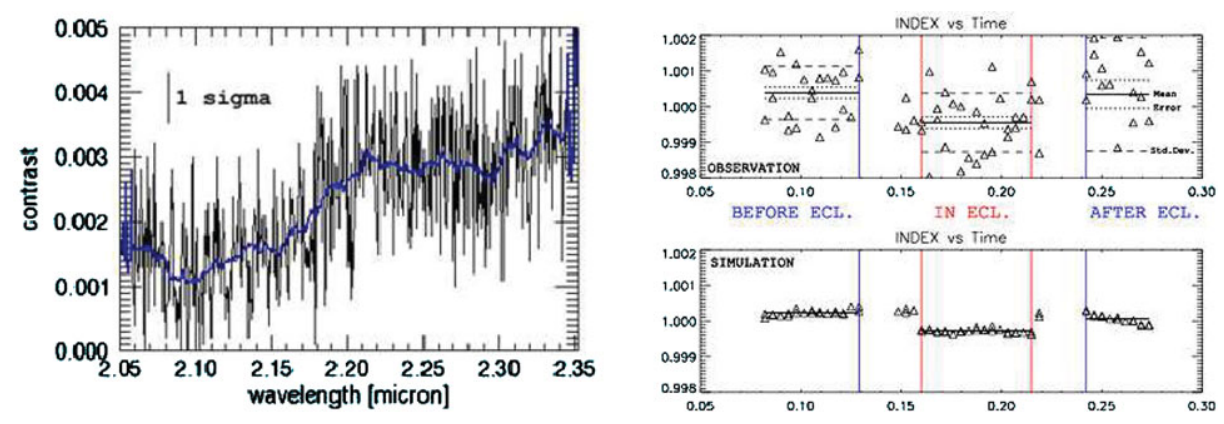

Figure 6. Right:K-Band contrast-spectra of 2005 HD209458b observation (Angerhausen 2006), (Angerhausen 2007), 30-channel smoothed signal (blue/thick). The residual OH lines have been corrected. The narrow band signal to noise ratios per sample position are of the order of 1250 in the $\mathrm{K}$ band spectrum. Left: final lightcurve of the spectral index of the 2.3-micron feature of the observed data (top; Phase-mean: solid, standard deviation: dashed, error: dotted) and a simulated dataset (bottom).

\section{References}

Angerhausen, D. et al. 2006, in: McLean, Ian S.; Iye, Masanori (eds.), Ground-based and Airborne Instrumentation for Astronomy, Proc. of the SPIE, 6269, 62694S

Angerhausen, D. et al. 2007, in: C. Afonso, D. Weldrake, \& Th. Henning (eds.), Transiting Extrasolar Planets Workshop, ASP Conference Series, 366, 262

Arribas, S. et al. 2006, PASP, 118, 839, 21-36

Barman, T. 2005, ApJ, 632, 1132-1139 